\title{
Titanium (Ti) Alloy Electron Beam (EB) Weld Fracture Surface Interpretation
}

\author{
Steven J. Gentz ${ }^{1}$ \\ 1. NASA, Langley Research Center, Hampton, VA
}

Failure analysis can be defined as collecting, interpreting, and documenting information to determine the failure cause. Data collection and interpretation should be a balance of: visual and microscopy; macro-, micro-, and nano-chemistry; and research and discussion. The following example centers on restrain in concluding a failure cause without evaluating all of the available data.

A Ti alloy pressure vessel designed to contain an inert gas was undergoing qualification testing. This vessel consisted of a cylindrical center section and hemispherical domes joined with circumferential EB welds. Pressurization/depressurization was through a fitting EB welded on one dome. The qualification test regime consisted of proof testing, nondestructive examination (NDE), several hundred pressure cycles to the maximum design pressure (MDP), and then pneumatic burst testing. The post proof NDE did not report any detectable defects, the cyclic testing occurred without incident, and the burst pressure was greater than two times the MDP. Following burst testing, the vessel was subjected to metallurgical analysis to examine the fracture surface and remnant hardware for anomalous features.

The vessel separated at a dome/cylinder EB weld. The cylindrical section showed outer diameter (OD) axial strain lines and diametric growth (i.e., barreling) plastic deformation. The fractured EB weld showed good tracking and uniformity. Housing wall thickness measurements in the cylindrical section adjacent to the weld fusion zone did not detect deviations from the drawing dimension.

The fracture surface presented three distinct zones, which were labeled the initial fracture (Figure 1), fast fracture, and final overload. The initial fracture was approximately $6.5 \mathrm{~cm}$ long and centered at the EB cover pass overlap. When viewed with a stereomicroscope, this zone was distinctly different from the adjacent fast fracture region, had an OD shear lip, and numerous tear ridges converging at multiple inner diameter (ID) locations. In addition, a demarcation line was seen at approximately mid span through the thickness. Depending on the light source and viewing angle, this boundary line appeared to turn towards the ID surface. As the vessel was subjected to repeated pressure cycles, this region was suspected to be a fatigue crack approximately $6.5 \mathrm{~cm}$ long by $0.3 \mathrm{~cm}$ deep. The project manager and structural analyst received this preliminary observation with alarm and skepticism as a defect of this magnitude should have failed well before the recorded burst pressure.

Subsequently the fracture surface was examined with electron microscopy and sectioned for metallography. Microscopy determined the preponderance of crack extension in this zone was a combination of ductile dimple and cleavage fracture with no evidence of organized cyclic fatigue. Metallography (Figure 2) determined the reported OD shear lip was associated with the EB cover pass fusion zone, and the demarcation line was a microstructural thermal effect from the cover pass. Figure 3 shows a composite of the metallography section compared with the corresponding location on the matting fracture surface.

Although the fracture exhibited unique characteristics, providing premature observations without sufficient data can be counterproductive as they may generate unnecessary confusion. 


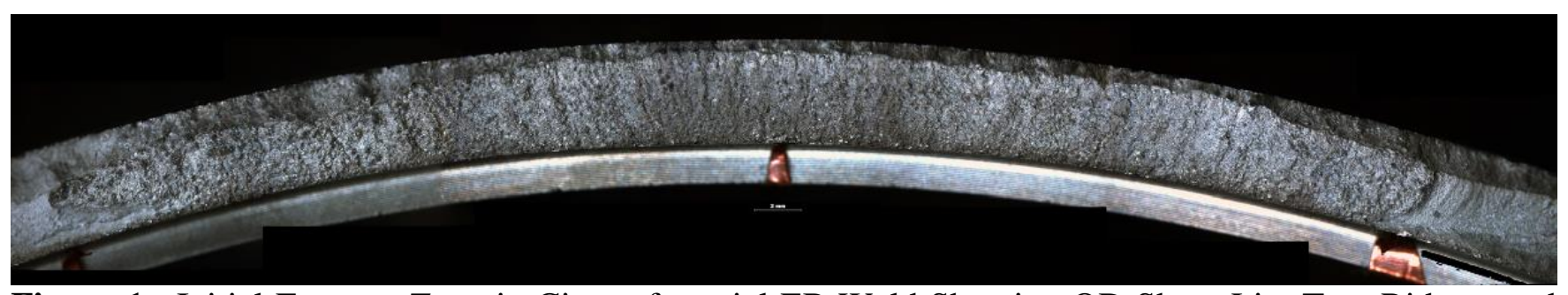

Figure 1. Initial Fracture Zone in Circumferential EB Weld Showing OD Shear Lip, Tear Ridges, and Mid Span Demarcation Line (3x magnification)

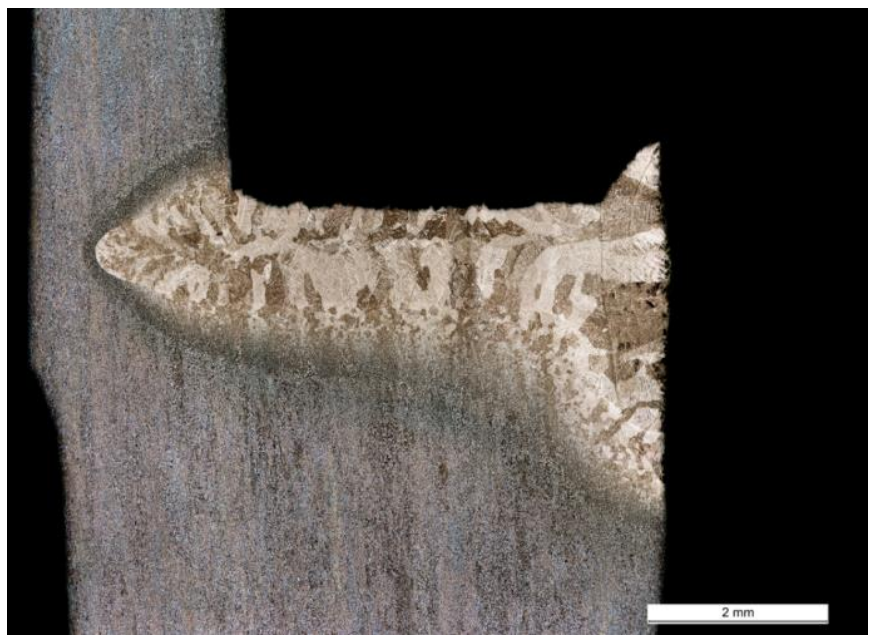

Figure 2. EB Weld Fracture Cross Section (10x magnification, etchant - Kroll's reagent)
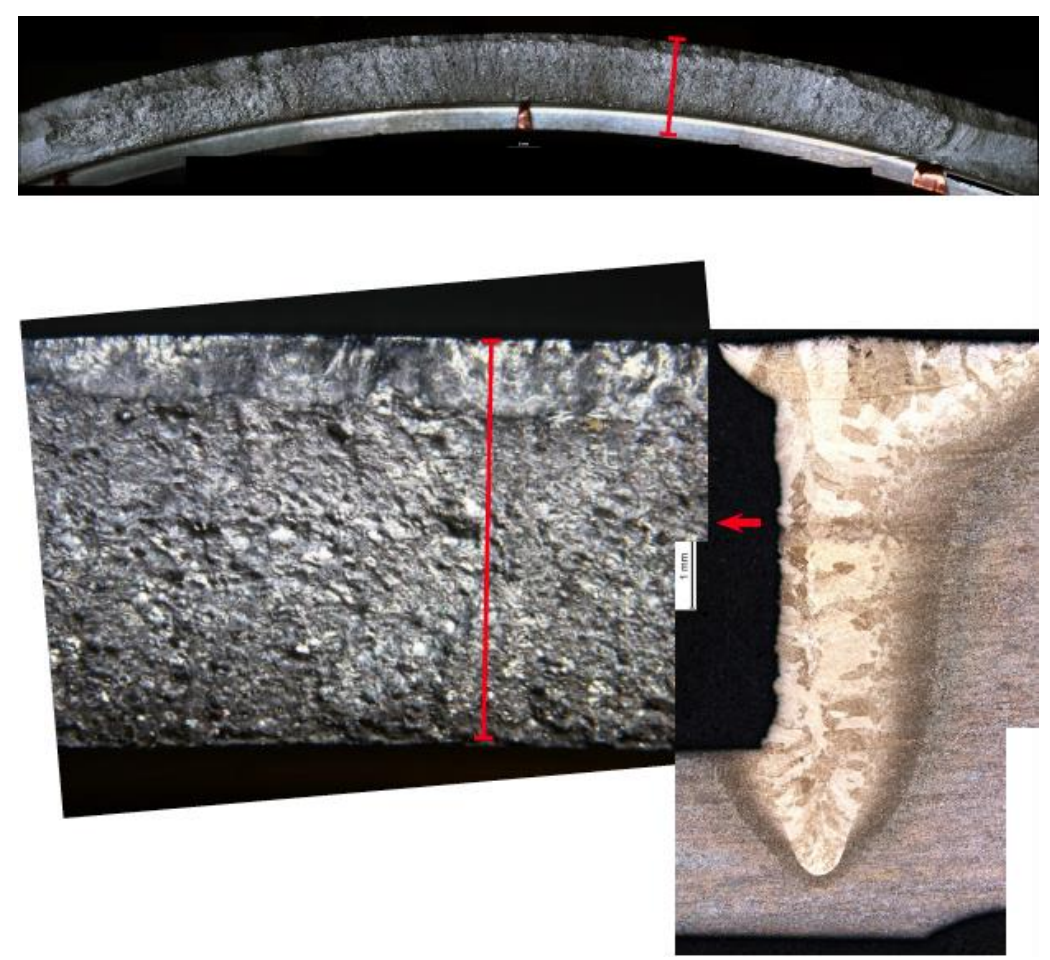

Figure 3. Composite of Optical, Electron Microscopy, and Metallographic Images Showing Relationship of Demarcation Line to Microstructural Feature (line indicates sectioning location) 